\title{
QT Dispersion Has No Prognostic Value in Patients with Symptomatic Heart Failure: An ELITE II Substudy
}

\author{
YI GANG, * TAKUYA ONO, * KATERINA HNATKOVA, * KENICH HASHIMOTO, * A. JOHN \\ CAMM ${ }^{*}$ BERTRAM PITT,† PHILIP A. POOLE-WILSON,‡ and MAREK MALIK* ON BEHALF OF \\ THE ELITE II INVESTIGATORS \\ From the *Department of Cardiological Sciences, St. George’s Hospital Medical School, London, \\ †University of Michigan Medical Center, Ann Arbor, Michigan, and the ¥Imperial College School of Medicine, \\ London, the United Kingdom
}

GANG, Y., ET AL.: QT Dispersion Has No Prognostic Value in Patients with Symptomatic Heart Failure: An ELITE II Substudy. This study prospectively investigated 3,118 standard 12-lead ECGs recorded in 1,804 patients, who participated in the Losartan Heart Failure Survival Study-ELITE II clinical trial. After exclusion of patients with rhythms other than sinus, or atrioventricular block, or poor quality ECGs, 986 (703 men, mean age $71 \pm 7$ years) with baseline ECGs were retained, of whom 615 patients had follow-up ECGs 4 months after randomization. QT intervals were manually measured with a digitizing board. Heart rate, QRS duration, maximum QT and JT intervals, QT and JT dispersion (the interval ranges across all measurable ECG leads) were analyzed. In the overall population, there were $140(14 \%)$ deaths from all causes, including 119 (12\%) cardiac and 59 (6\%) sudden deaths during a follow-up of $540 \pm 153$ days. The mean heart rate was significantly faster in nonsurvivors than in survivors ( $77 \pm 16$ vs $74 \pm 14$ beats/min, $P=0.006)$, and in patients who died of cardiac death (76 \pm 16 beats $/ \mathrm{min}, P=0.04$ vs survivors). Mean QRS duration was significantly longer in nonsurvivors (107 $\pm 25 \mathrm{~ms})$, and in the subgroups who died of cardiac (107 $\pm 24 \mathrm{~ms}$ ) or sudden death (112 $\pm 23 \mathrm{~ms})$ than in survivors (99 $\pm 24 \mathrm{~ms}, P<0.01$ for all). The maximum and corrected (QTC) QT intervals were similar for nonsurvivors, regardless of cause of death, and in survivors ( $P=N S$ for all comparisons). Significantly shorter maximum and corrected (JTC) $J T$ intervals were observed in victims of any mode of death compared to survivors $(P<0.05$ for all). There was no significant difference in QT or JT dispersion between patients with any mode of death and survivors $(P>0.1$ for all). Neither losartan nor captopril significantly modified QT or JT dispersion. In conclusion, increased QT dispersion is not associated with increased mortality in patients with heart failure, and is not suitable to examine drug efficacy in these patients. (PACE 2003; 26[Pt. II]:394-400)

QT dispersion, heart failure, cardiac death, sudden death, losartan, captopril

\section{Introduction}

Despite advances in pharmaceutical and electronic device therapies, mortality remains high in patients with heart failure. Therefore, it is important to identify patients who are at high risk of cardiac death, sudden cardiac death in particular.

QT dispersion was originally proposed as a simple noninvasive technique for quantifying nonspecific repolarization abnormalities and as a marker of vulnerability to ventricular arrhythmias. ${ }^{1}$ Several early studies suggested that QT dispersion predicted malignant ventricular arrhythmias and mortality in patients with various car-

Supported in part by an Education Grant of Merck Research Laboratories.

Address for reprints: Yi Gang, M.D., Ph.D., Dept. of Cardiological Sciences, St. George's Hospital Medical School, Cranmer Terrace, London SW17 0RE, United Kingdom. Fax: +44-208725-0846; e-mail: y.gang@sghms.ac.uk diac diseases. ${ }^{2-6}$ More recent studies did not confirm these results. ${ }^{7-9}$ The early studies reporting that increased QT dispersion identified patients at risk of sudden death or cardiac mortality included patients with heart failure..$^{2,4,10,11}$

Based on these observations, a QT dispersion substudy was prospectively included in the protocol of the ELITE II trial, and was designed to evaluate the prognostic value of QT dispersion and the effect of treatment in a large number of well-characterized elderly patients with heart failure.

\section{Patient Population}

\section{Patients and Methods}

The patient population was chosen from the Losartan Heart Failure Survival Study-ELITE II clinical trial. ${ }^{12}$ Briefly, the ELITE II was a randomized, double-blind clinical trial conducted at 289 sites in 46 countries from June 1997 to May 1998. The study enrolled 3,152 patients aged $\geq 60$ years 
(85\% $\geq 65$ years) in New York Heart Association (NYHA) functional Classes II-IV, and with a left ventricular ejection fraction $\leq 0.40$. All study patients were regularly followed at study centers.

Patients included into this substudy were followed up for a mean of $540 \pm 153$ days. The endpoints included all-cause, cardiac, and sudden cardiac mortality classified by the event committee of the trial. The clinical data were unknown to the investigators of the QT dispersion substudy before all measurements and calculations were completed.

\section{Electrocardiographic Recordings}

A total of 3,118 standard 12-lead electrocardiograms (ECGs) recorded in 1,804 of the 3,152 patients enrolled in ELITE II were available for central evaluation. All 1,804 patients had a baseline ECG recorded before randomization, and 1,314 had follow-up ECGs recorded at 4 months after randomization. A single investigator blinded to the patients' characteristics and clinical outcomes analyzed all ECGs and made the QT measurements. ECGs with atrial fibrillation $(n=412)$, rhythms other than sinus $(n=82)$, second-degree atrioventricular heart block or higher, frequent premature complexes $(\mathrm{n}=34)$, inconspicuous $\mathrm{T}$ waves $(\mathrm{n}=$ 65 ), and tracings of poor quality or with $<4$ analyzable leads $(n=522)$ were excluded from the analysis. In addition, 402 follow-up ECGs were excluded because the corresponding baseline ECG was not analyzable. Ultimately, 986 baseline and 615 follow-up ECGs were analyzed.

The ECGs were available as paper printouts recorded with various instrumentation used at each individual centers. Most tracings were standard 10-second recordings of four consecutive sets of three leads recorded simultaneously, a minority were simultaneous 12 or $2 \times 6$-lead recordings, and a few consisted of separate single lead recordings. The majority of ECGs were recorded at a paper speed of $25 \mathrm{~mm} / \mathrm{s}$, and the remainder at $50 \mathrm{~mm} / \mathrm{s}$.

\section{ECG Measurements}

In each ECG lead, the onset of the QRS complex, J point, and the offset of the $\mathrm{T}$ wave were measured from two consecutive cardiac cycles whenever possible, and averaged values were used for interval analysis. QT intervals were measured from the onset of the QRS complex to the end of repolarization, defined as the return to the baseline, or the nadir between the $\mathrm{T}$ and physiological U wave, when applicable. The J point was defined as the junction of the $S$ wave and the ST segment. When the end of repolarization could not be ascertained, the measurement in that lead was not made. At least four measurable leads were required for the inclusion of an ECG into the study.

All measurements were performed manually using a digitizing board (resolution $0.025 \mathrm{~mm}$, Cherry, Harpender, United Kingdom) driven by a purpose-built interactive software. An customdesigned program was used for on-line quality control of the measurement and to calculate QT interval duration and dispersion. To eliminate the confounding factor of bundle branch block, JT interval and JT dispersion were also derived. QT and JT dispersion was defined as the range of QT and JT intervals across all measurable ECG leads, respectively.

\section{Data Analysis and Statistics}

ECG variables measured included heart rate, QRS duration, maximum QT and JT intervals, and heart rate corrected QT and JT intervals (QTc, JTc by Fridericia's formula), and QT and JT dispersion. Heart rate QTc or JTc dispersion was not used. ${ }^{13,14}$ No attempt was made to correct dispersion values for missing leads. ${ }^{15}$

Univariate comparisons between groups were made by chi-square test for categorical data and two-sample $t$-test for continuous data, while a paired $t$-test was used for comparison of the measurements before and after treatment. Univariate relation between baseline variables and mortality was assessed by the Cox proportional hazards model with relative risk derived. Multivariate Cox analysis considered any variables that showed univariate association with endpoints. Dichotomized data were used in regression models and the mean values of all studied patients were used as dichotomy limits. Kaplan-Meier survival analysis was performed and differences between survival probabilities were compared using log-rank test. Following a prospectively defined protocol of the substudy, patients were stratified by a dichotomy of QT dispersion of $80 \mathrm{~ms}$. Continuous data are presented as mean \pm SD. A P value $<0.05$ was considered statistically significant.

\section{Results \\ Clinical Characteristics and Outcomes}

The clinical characteristics of the study population are presented in Table I. There were no significant differences in clinical characteristics between patients with and without QT dispersion $>80 \mathrm{~ms}$ with the exception of more patients with QT dispersion $>80 \mathrm{~ms}$ having QRS duration $>120 \mathrm{~ms}(\mathrm{P}=0.02)$.

Study patients were followed for a mean of $540 \pm 153$ days (range 7-780 days). During followup, there were 140 (14\%) deaths from all causes, 
Table I.

Clinical Characteristics of Study Patients Before Randomization

\begin{tabular}{|c|c|c|c|}
\hline Characteristics & All Patients $(n=986)$ & QTd $>80 \mathrm{~ms}(\mathrm{n}=540)$ & $\mathrm{QTd} \leq 80 \mathrm{~ms}(\mathrm{n}=446)$ \\
\hline Age (years) & $71.2 \pm 6.9$ & $71.3 \pm 6.9$ & $70.9 \pm 6.8$ \\
\hline Sex (men) & $703(71 \%)$ & $392(73 \%)$ & $311(70 \%)$ \\
\hline Duration of heart failure (months) & $27.3 \pm 38.9$ & $29.0 \pm 42.0$ & $25.2 \pm 34.7$ \\
\hline NYHA functional Class III/IV & $453(46 \%)$ & 237 (44\%) & $216(48 \%)$ \\
\hline Left ventricular ejection fraction (\%) & $31.3 \pm 6.9$ & $31.3 \pm 6.9$ & $31.2 \pm 7.0$ \\
\hline QRS duration $>120 \mathrm{~ms}$ & $216(22 \%)$ & $134(25 \%) *$ & $82(18 \%)$ \\
\hline \multicolumn{4}{|l|}{ Medical history } \\
\hline Ischemic heart disease & $769(78 \%)$ & $421(78 \%)$ & $348(78 \%)$ \\
\hline Diabetes & $223(23 \%)$ & $121(22 \%)$ & $102(23 \%)$ \\
\hline \multicolumn{4}{|l|}{ Mèdications } \\
\hline Losartan & $487(49 \%)$ & $259(48 \%)$ & $228(51 \%)$ \\
\hline Captopril & $499(51 \%)$ & $281(52 \%)$ & $218(49 \%)$ \\
\hline$\beta$-blockers & $230(23 \%)$ & $123(23 \%)$ & $107(24 \%)$ \\
\hline \multicolumn{4}{|l|}{ Laboratory results } \\
\hline Serum potassium (mmol/L) & $4.4 \pm 0.5$ & $4.4 \pm 0.5$ & $4.4 \pm 0.5$ \\
\hline Serum sodium (mmol/L) & $140.5 \pm 3.5$ & $140.7 \pm 3.6$ & $140.4 \pm 3.4$ \\
\hline Serum creatinine ( $\mu \mathrm{mol} / \mathrm{L})$ & $103.6 \pm 27.5$ & $104.4 \pm 27.9$ & $102.6 \pm 27.0$ \\
\hline
\end{tabular}

${ }^{*} \mathrm{P}=0.02$ for comparison between patients with and without $\mathrm{QT}$ dispersion $>80 \mathrm{~ms}$; NYHA $=$ New York Heart Association, $\mathrm{QTd}=\mathrm{QT}$ dispersion.

including $119(12 \%)$ cardiac and $59(6 \%)$ sudden deaths.

\section{ECG Variables}

Conventional ECG measurements are shown in Table II. Significant differences in heart rate were found between survivors and nonsurvivors and between survivors and the subgroup who died of cardiac death, but not of sudden death. Mean QRS duration was significantly longer in patients with all-cause mortality, cardiac death, or sudden death compared with survivors. Paradoxically, sig- nificantly shorter maximum JT and JTc intervals were observed in victims of all-cause or cardiac deaths than in survivors.

The mean QT and JT dispersion in all patients were $86 \pm 31 \mathrm{~ms}(8-190 \mathrm{~ms})$ and $82 \pm 32 \mathrm{~ms}(9$ $181 \mathrm{~ms})$, respectively. There were $540(55 \%)$ patients with QT dispersion $>80 \mathrm{~ms}$, and 479 (49\%) patients with JT dispersion $>80 \mathrm{~ms}$.

In patients with $\mathrm{QRS} \geq 120 \mathrm{~ms}(\mathrm{n}=216)$, both QT dispersion and JT dispersion tended to be greater than in those with QRS $<120 \mathrm{~ms}$ (90 \pm 29 vs $85 \pm 32 \mathrm{~ms}, \mathrm{P}=0.055$, and $84 \pm 30$ vs $82 \pm$ $33 \mathrm{~ms}, \mathrm{P}=\mathrm{NS}$, respectively).

Table II.

Measurements of Conventional ECG Parameters at Baseline

\begin{tabular}{lccccc}
\hline & $\begin{array}{c}\text { All Patients } \\
(\mathbf{n = 9 8 6 )}\end{array}$ & $\begin{array}{c}\text { All Death } \\
\mathbf{( n = 1 4 0 )}\end{array}$ & $\begin{array}{c}\text { Cardiac Death } \\
(\mathbf{n = 1 1 9 )}\end{array}$ & $\begin{array}{c}\text { Sudden Death } \\
(\mathbf{n}=\mathbf{5 9})\end{array}$ & $\begin{array}{c}\text { Survivors } \\
(\mathbf{n}=\mathbf{8 4 6})\end{array}$ \\
\hline Heart rate (beats/min) & $74 \pm 15$ & $77 \pm 16^{*}$ & $76 \pm 16+$ & $76 \pm 16$ & $74 \pm 14$ \\
Mean QRS duration (ms) & $100 \pm 24$ & $107 \pm 25 \ddagger$ & $107 \pm 24 \ddagger$ & $112 \pm 23 \ddagger$ & $99 \pm 24$ \\
Maximum QT interval (ms) & $427 \pm 49$ & $423 \pm 50$ & $424 \pm 49$ & $433 \pm 49$ & $428 \pm 48$ \\
Maximum QTc interval (ms) & $454 \pm 41$ & $455 \pm 41$ & $455 \pm 41$ & $464 \pm 39$ & $454 \pm 41$ \\
Maximum JT interval (ms) & $326 \pm 46$ & $315 \pm 47^{*}$ & $314 \pm 45^{*}$ & $319 \pm 47$ & $327 \pm 45$ \\
Maximum JTc interval (ms) & $346 \pm 38$ & $338 \pm 37+$ & $337 \pm 36^{*}$ & $342 \pm 37$ & $347 \pm 38$ \\
\hline
\end{tabular}

${ }^{*} \mathrm{P} \leq 0.01 ;+\mathrm{P}<0.05 ; \neq \mathrm{P} \leq 0.001$ for comparison with survivors. $E C G=$ electrocardiogram. 
QT DISPERSION IN HEART FAILURE

Table III.

Univariate Risk Analysis

\begin{tabular}{|c|c|c|c|c|c|c|}
\hline \multirow[b]{2}{*}{ Variables } & \multicolumn{2}{|c|}{ All-Cause Death } & \multicolumn{2}{|c|}{ Cardiac Death } & \multicolumn{2}{|c|}{ Sudden Death } \\
\hline & RR (Cl) & $\mathbf{P}$ & $\mathrm{RR}(\mathrm{Cl})$ & $\mathbf{P}$ & RR (Cl) & $\mathbf{P}$ \\
\hline Age $>71$ years & $1.29(0.93-1.80)$ & NS & $1.12(0.78-1.61)$ & NS & $0.99(0.59-1.66)$ & NS \\
\hline Sex (men) & $1.33(0.90-1.96)$ & NS & 1.54 (0.99-2.39) & NS & $1.34(0.74-2.45)$ & NS \\
\hline Weight $<72$ kg & $1.28(0.91-1.78)$ & NS & $1.20(0.84-1.72)$ & NS & $1.14(0.68-1.90)$ & NS \\
\hline Duration of etiology $>27$ months & $1.60(1.15-2.24)$ & 0.006 & $1.71(1.19-2.46)$ & 0.004 & $1.49(0.88-2.51)$ & NS \\
\hline Heart rate $>74$ beats $/ \mathrm{min}$ & $1.28(0.92-1.79)$ & NS & $1.24(0.86-1.77)$ & NS & $1.35(0.81-2.25)$ & NS \\
\hline Mean QRS duration >100 ms & $1.99(1.43-2.78)$ & $<0.001$ & $2.13(1.48-3.07)$ & $<0.001$ & $3.10(1.81-5.31)$ & $<0.001$ \\
\hline Maximum QT interval $>427 \mathrm{~ms}$ & $0.84(0.60-1.18)$ & & $0.86(0.60-1.23)$ & & $1.10(0.66-1.83)$ & NS \\
\hline Maximum QTc interval >454 ms & $0.98(0.70-1.37)$ & NS & $0.92(0.64-1.32)$ & NS & $1.29(0.78-2.16)$ & NS \\
\hline Maximum JT interval $<326 \mathrm{~ms}$ & $1.46(1.04-2.06)$ & 0.029 & $1.56(1.08-2.27)$ & 0.019 & $1.20(0.72-2.00)$ & NS \\
\hline Maximum JTc interval <346 ms & $1.32(0.94-1.85)$ & NS & $1.44(0.99-2.08)$ & NS & $1.30(0.77-2.19)$ & NS \\
\hline QT dispersion > $80 \mathrm{~ms}$ & $0.81(0.58-1.13)$ & NS & $0.80(0.56-1.14)$ & NS & $1.07(0.64-1.80)$ & NS \\
\hline JT dispersion $>80 \mathrm{~ms}$ & $0.92(0.66-1.28)$ & NS & $0.86(0.60-1.24)$ & NS & $1.39(0.83-2.33)$ & NS \\
\hline NYHA functional class III-IV & $2.14(1.52-3.02)$ & $<0.001$ & $2.24(1.54-3.26)$ & $<0.001$ & $1.91(1.14-3.22)$ & 0.014 \\
\hline LV ejection fraction $<25 \%$ & $1.59(1.08-2.34)$ & 0.019 & $1.61(1.06-2.44)$ & 0.026 & $1.57(0.86-2.85)$ & NS \\
\hline Serum sodium $<$ low limits & $0.85(0.37-1.92)$ & NS & $0.82(0.34-2.02)$ & NS & $0.63(0.16-2.59)$ & NS \\
\hline Serum potassium > upper limits & $1.95(1.14-3.33)$ & 0.015 & $2.14(1.23-3.74)$ & 0.008 & 2.24 (1.02-4.92) & 0.046 \\
\hline Serum creatinine $>$ upper limits & $1.43(0.98-2.09)$ & NS & $1.57(1.05-2.35)$ & 0.027 & $1.54(0.87-2.73)$ & NS \\
\hline
\end{tabular}

$\mathrm{Cl}=95 \%$ confidence interval; $\mathrm{LV}=$ left ventricular; $\mathrm{NS}=$ not statistically significant; NYHA = New York Heart Association;

$\mathrm{RR}=$ relative risk.

No significant differences in QT dispersion or JT dispersion were found between patients who were and were not on $\beta$-adrenergic blocker therapy at randomization ( $88 \pm 31$ vs $85 \pm 31 \mathrm{~ms}, \mathrm{P}=$ NS; $84 \pm 31$ vs $82 \pm 32 \mathrm{~ms}, \mathrm{P}=\mathrm{NS}$, respectively). No significant differences in QT dispersion were found between all nonsurvivors ( $84 \pm 29 \mathrm{~ms}$ ), the subgroups who died of cardiac death $(83 \pm 28 \mathrm{~ms})$ or sudden death ( $86 \pm 28 \mathrm{~ms}$ ), and survivors ( $86 \pm$ $31 \mathrm{~ms})(\mathrm{P}=\mathrm{NS}$ for all). Similar results were found in JT dispersion $(80 \pm 32,78 \pm 30,83 \pm 33$ vs $83 \pm 32 \mathrm{~ms}$, respectively; $\mathrm{P}=\mathrm{NS}$ for all).

Of 846 survivors, 470 (56\%) had a QT dispersion $>80 \mathrm{~ms}$. Among patients who died from all causes, from cardiac death, or from sudden death, there were $70(50 \%), 59(50 \%)$, and 34 (58\%) patients who had a QT dispersion $>80 \mathrm{~ms}$, respectively ( $\mathrm{P}>0.2$ for all).

\section{Survival Analysis}

Among all risk factors analyzed, six variables were significantly associated with all-cause mortality in univariate analysis (Table III), and seven with cardiac death. Only a mean QRS duration $>100 \mathrm{~ms}$, NYHA functional Class III-IV, and serum potassium above the normal upper limit were associated with sudden death. Increased QT disper- sion and JT dispersion ( $>80 \mathrm{~ms}$ ) were not associated with any mode of death.

In the multivariate analyses (Table IV), NYHA functional class, mean QRS duration, hyperkalemia, and duration of disease remained independent predictors of all-cause mortality and cardiac mortality, while mean QRS duration, NYHA functional class, and serum potassium predicted sudden death independently of other variables.

There was no significant difference in survival rate between patient groups stratified by QT dispersion of $80 \mathrm{~ms}$ (Fig. 1).

\section{Effect of Treatment on Ventricular Repolarization Dispersion}

The effects of losartan and captopril on ventricular repolarization dispersion are shown in Table V. QT dispersion and JT dispersion were similar in patients on either medication, and before and during treatment. No survival difference was found among patients with QT dispersion $>80 \mathrm{~ms}$ (or JT dispersion $>80 \mathrm{~ms}$ ) when treated with losartan versus captopril (Fig. 2).

\section{Discussion}

This study found no prognostic value of QT dispersion or JT dispersion with respect to any mode of death in the subpopulation of ELITE II, 
Table IV.

Multivariate Analysis of the Relation Between Study Variables and Deaths

\begin{tabular}{|c|c|c|c|c|c|c|}
\hline \multirow[b]{2}{*}{ Variables } & \multicolumn{2}{|c|}{ All-Cause Death } & \multicolumn{2}{|c|}{ Cardiac Death } & \multicolumn{2}{|c|}{ Sudden Death } \\
\hline & $\mathrm{RR}(\mathrm{Cl})$ & $\mathbf{P}$ & $\mathrm{RR}(\mathrm{Cl})$ & $\mathbf{P}$ & $\mathrm{RR}(\mathrm{Cl})$ & $\mathbf{P}$ \\
\hline Duration of etiology $>27$ months & $1.45(1.03-2.03)$ & 0.033 & $1.50(1.04-2.16)$ & 0.031 & - & - \\
\hline Mean QRS duration >100 ms & $1.81(1.29-2.54)$ & 0.001 & $1.91(1.32-2.76)$ & 0.001 & $3.09(1.80-5.31)$ & $<0.001$ \\
\hline NYHA functional Class III-IV & $1.90(1.34-2.70)$ & $<0.001$ & $1.98(1.35-2.89)$ & $<0.001$ & $1.84(1.09-3.09)$ & 0.022 \\
\hline Serum potassium $>$ upper limits & $1.96(1.14-3.37)$ & 0.015 & $2.06(1.16-3.62)$ & 0.013 & $2.49(1.13-5.48)$ & 0.024 \\
\hline
\end{tabular}

$\mathrm{Cl}=95 \%$ confidence interval; $\mathrm{LV}=$ left ventricular; NYHA = New York Heart Association; RR = relative risk.

while some other conventional ECG measurements were predictive of mortality. A prospectively defined dichotomy of QT dispersion $>80 \mathrm{~ms}$ failed to stratify for an increase in risk of death, or to show survival differences between losartan and captopril treatment. Normal values of QT dispersion have never been established and have been reported to vary widely between 10 and $71 \mathrm{~ms}$, mostly between 30 and $60 \mathrm{~ms}$ in early studies. ${ }^{16,17}$ When this substudy was designed, a value of QT dispersion $>80$ ms was generally considered to be beyond the "normal limit." QT dispersion $>80 \mathrm{~ms}$ was also reported to be an independent predictor of sudden death and arrhythmic events in a study of a smaller population. ${ }^{4}$ Presently, it seems arbitrary and futile to set a dichotomy point for "abnormal” QT dispersion in prospective studies.

Since excluding patients with bundle branch block may have created a selection bias, they were included in the QT measurement and analysis. Moreover, some studies have suggested that QT dispersion was not influenced by the presence or absence of bundle branch block. ${ }^{8,9}$ In the current study, the negative results obtained with QT dispersion were reproduced with JT dispersion.

Surprisingly, the study found significantly shorter JT and JTc intervals in nonsurvivors. This finding may be related to the fact that they had a longer mean QRS duration. A shortened JT or JTc interval is unlikely to be an independent prognostic marker in heart failure as shown by this multivariate analysis.

\section{QT Dispersion in Heart Failure}

In the present study QT dispersion appeared greater $(86 \pm 31 \mathrm{~ms})$ than that reported in normal subjects. ${ }^{17}$ A mean QT dispersion (with the same system for manual measurement) of $42 \pm$ $13 \mathrm{~ms}^{18}$ and $44 \pm 16 \mathrm{~ms}^{19}$ was previously observed in the authors' laboratory in normal ECGs. Despite several methodological problems, QT dispersion values found in the present study were similar to those reported by others in patients with heart failure $^{5,8,9,11}$ The results from the present study regarding the prognostic value of QT dispersion are at variance with early reports..$^{2,4,5,10,11}$

Table V.

Comparison of QT Dispersion and Other ECG Measurements Between Losartan- and Captopril-Treated Patients

\begin{tabular}{|c|c|c|c|c|c|c|c|}
\hline & \multicolumn{3}{|c|}{ Losartan $(n=299)$} & \multicolumn{3}{|c|}{ Captopril $(n=316)$} & \multirow[b]{2}{*}{${ }^{*} \mathbf{P}$} \\
\hline & Baseline & At 4 months & $\mathbf{P}$ & Baseline & At 4 months & $\mathbf{P}$ & \\
\hline Heart rate (beats/min) & $75 \pm 15$ & $72 \pm 13$ & 0.000 & $74 \pm 15$ & $72 \pm 14$ & 0.02 & 0.80 \\
\hline Maximum QT interval (ms) & $425 \pm 50$ & $426 \pm 45$ & 0.59 & $430 \pm 50$ & $426 \pm 48$ & 0.06 & 0.93 \\
\hline Maximum JT interval (ms) & $324 \pm 45$ & $325 \pm 41$ & 0.79 & $328 \pm 48$ & $324 \pm 43$ & 0.16 & 0.80 \\
\hline tMaximum QTc interval (ms) & $453 \pm 41$ & $449 \pm 39$ & 0.08 & $457 \pm 42$ & $449 \pm 42$ & 0.000 & 0.95 \\
\hline tMaximum JTc interval (ms) & $346 \pm 37$ & $342 \pm 35$ & 0.13 & $347 \pm 40$ & $341 \pm 35$ & 0.007 & 0.71 \\
\hline QT dispersion (ms) & $85 \pm 29$ & $86 \pm 31$ & 0.84 & $88 \pm 32$ & $84 \pm 32$ & 0.09 & 0.52 \\
\hline JT dispersion (ms) & $83 \pm 32$ & $84 \pm 32$ & 0.77 & $84 \pm 33$ & $81 \pm 31$ & 0.20 & 0.23 \\
\hline
\end{tabular}

${ }^{*} \mathrm{P}$ values for comparison between medication groups at 4 months after randomization. + Fridericial formula was used for heart rate corrected QT and JT intervals. ECG = electrocardiogram; JTc = corrected JT interval; QTc = corrected QT interval. 


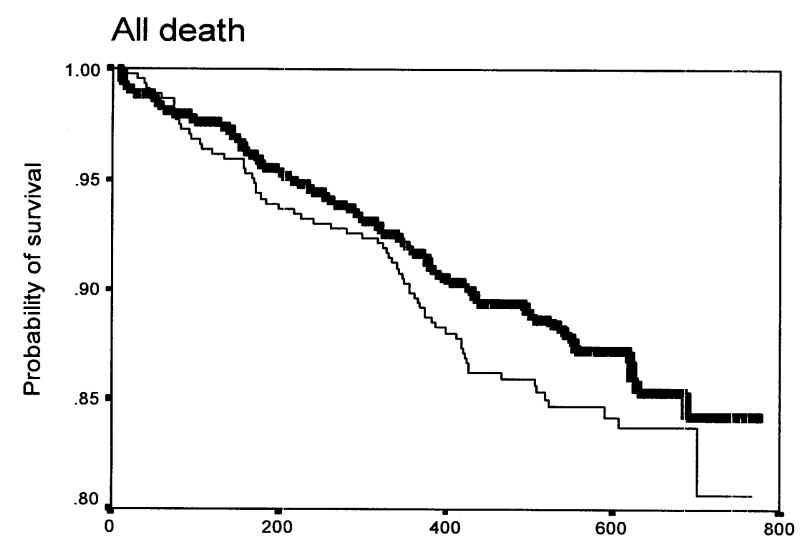

Cardiac death
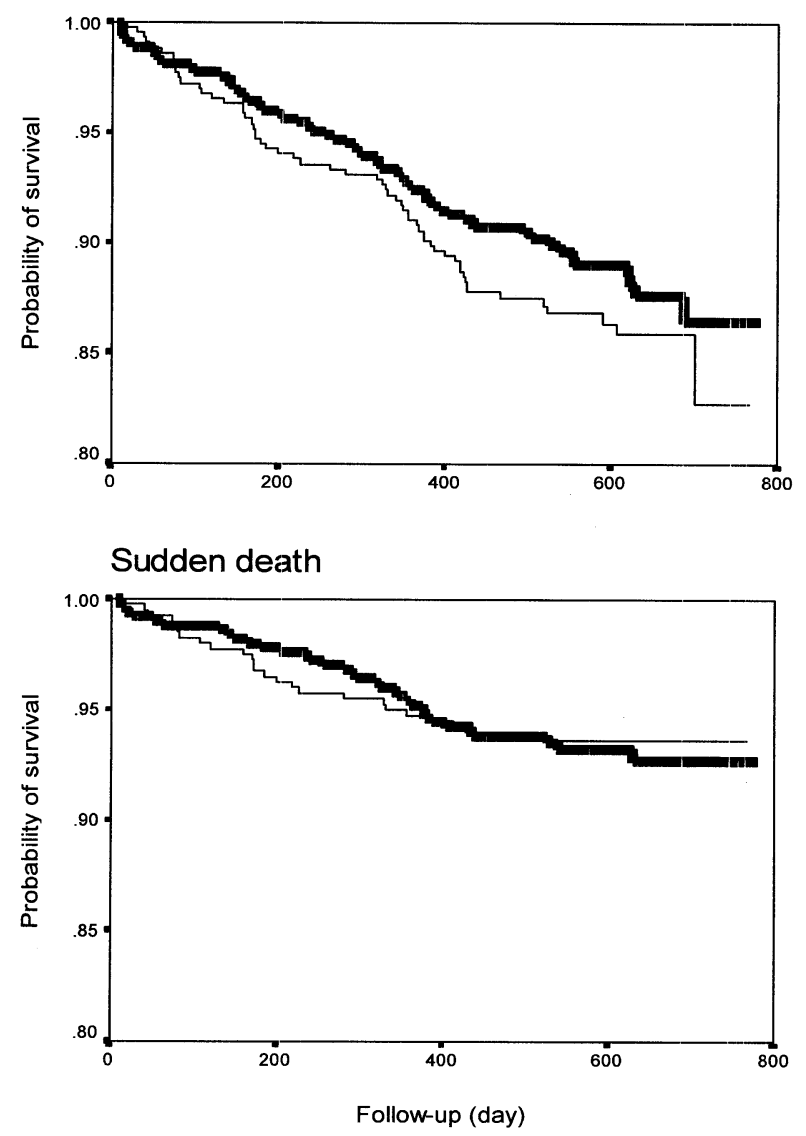

Figure 1. Survival curves of all study patients $(n=986)$ stratified by $Q T$ dispersion $>80$ ms for all-cause mortality $(A)$, cardiac mortality $(B)$, and sudden cardiac mortality $(C)$. The thick lines indicate patients with $Q T$ dispersion $\leq 80 \mathrm{~ms}$, and the thin lines indicate patients with $Q T$ dispersion $>80 \mathrm{~ms}$ (log-rank test, $P=N S$ for all).

Galinier et al. ${ }^{4}$ reported that a QT dispersion $>80 \mathrm{~ms}$ independently predicted sudden death (RR 4.9, 95\% confidence interval [CI] 1.4-16.8, $\mathrm{P}<0.02)$ and arrhythmic events (RR 4.5, 95\% CI
Study Patients Stratified by QT Dispersion $>80 \mathrm{~ms}$

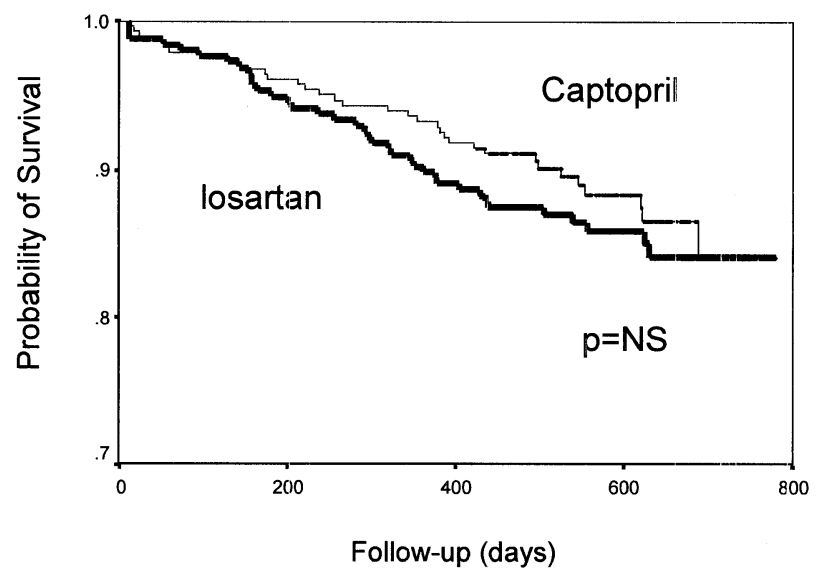

Figure 2. All-cause mortality-free curves of patients with QT dispersion $>80 \mathrm{~ms}$, stratified by medication. The bold line indicates losartan-treated patients, and thin line indicates captopril-treated patients.

1.5-13.5, $\mathrm{P}<0.01)$ in patients with dilated cardiomyopathy $(\mathrm{n}=119)$, though was not predictive in patients with ischemic heart disease $(\mathrm{n}=$ 86). ${ }^{4}$ In the present study, QT dispersion was associated with neither mortality nor underlying cause of heart failure. The findings that QT and JT dispersion does not have prognostic value in heart failure are consistent with more recent reports. ${ }^{8,9,20}$ In the Danish Investigations of Arrhythmia and Mortality on Dofetilide congestive heart failure (DiamondCHF) substudy, QT dispersion had no prognostic value with respect to all-cause mortality, cardiac mortality, or cardiac arrhythmic mortality in 703 patients with advanced congestive heart failure. ${ }^{9}$ Similarly, in the United Kingdom Heart Failure Evaluation and Assessment of Risk Trial (UKHEART) substudy ${ }^{8}$ none of the QT or JT parameters predicted outcome independently in 495 patients with mild or moderate heart failure.

A substudy of the former ELITE heart failure study showed that captopril, but not losartan, increased QT dispersion. ${ }^{21}$ However, this was not confirmed in this analysis. Losartan and captopril treatments both reduced heart rate significantly, but QT and JT dispersion remained practically unchanged.

\section{Study Limitations}

The study ECGs were not all recorded at the same paper speed and printed in a consistent format. Although some studies showed that paper speed influences the accuracy of measurements, ${ }^{22,23}$ this cannot fully explain the results from the current study since only a small proportion of ECGs were recorded at $50 \mathrm{~mm} / \mathrm{s}$. 
Theoretically, an accurate assessment of QT dispersion requires all 12 leads of the ECG to be recorded simultaneously to avoid the effect of QT dynamicity. However, the slow dynamicity of the QT interval makes QT dispersion measurement based on the simultaneous recording of six or three leads during ectopic-free sinus rhythm acceptable for practical purpose. ${ }^{16}$

\section{Study Implications}

This study demonstrates that the measurement of QT dispersion is unlikely to provide prognostic information in patients with heart failure, and confirms the findings of another multicenter QT dispersion substudy reported by Brendorp et al. ${ }^{9}$ While QT dispersion may approximate and remotely reflect nonspecific $\mathrm{T}$ wave changes,

\section{References}

1. Day CP, McComb JM, Campbell RW. QT dispersion: An indication of arrhythmia risk in patients with long QT intervals. Br Heart J 1990; 63:342-344.

2. Barr CS, Naas A, Freeman M, et al. QT dispersion and sudden unexpected death in chronic heart failure. Lancet 1994; 343:327329.

3. Perkiömäki JS, Koistinen J, Yli-Mäyry S, et al. Dispersion of QT interval in patients with and without susceptibility to ventricula tachyarrhythmias after previous myocardial infarction. J Am Coll Cardiol 1995; 26:174-179.

4. Galinier M, Vialette JC, Fourcade J, et al. QT interval dispersion as a predictor of arrhythmic events in congestive heart failure. Importance of aetiology. Eur Heart J 1998; 19:1054-1062.

5. Spargias KS, Lindsay SJ, Kawar GI, et al. QT dispersion as a predictor of long-term mortality in patients with acute myocardial infarction and clinical evidence of heart failure. Eur Heart J 1999; 20:1158-1165.

6. Okin PM, Devereux RB, Howard BV, et al. Assessment of QT interval and QT dispersion for prediction of all-cause and cardiovascular mortality in the American Indians. The Strong Heart Study. Circulation 2000; 101:61-66.

7. Zabel M, Klingenheben T, Franz MR, et al. Assessment of QT dispersion for prediction of mortality or arrhythmic events after myocardial infarction: Results of a prospective, long-term follow-up study. Circulation 1998; 97:2543-2550.

8. Brooksby P, Batin PD, Nolan J, et al. The relationship between QT intervals and mortality in ambulant patients with chronic heart failure. The United Kingdom heart failure evaluation and assessment of risk trial (UK-HEART). Eur Heart J 1999; 20:1335-1341.

9. Brendorp B, Elming H, Jun L, et al. QT dispersion has no prognostic information for patients with advanced congestive heart failure and reduced left ventricular systolic function. Circulation 2001; 103:831-835.

10. Fu GS, Meissner A, Simon R. Repolarization dispersion and sudden cardiac death in patients with impaired left ventricular function. Eur Heart J 1997; 18:281-289.

11. Pinsky DJ, Sciacca RR, Steinberg JS. QT dispersion as a marker of risk in patients awaiting heart transplantation. J Am Coll Cardiol 1997; 29:1576-1584.

12. Pitt B, Poole-Wilson P, Segal R, et al. Effects of losartan versus captopril on mortality in patients with symptomatic heart failure: Rationale, design, and baseline characteristics of patients in the Losartan Heart Failure Survival Study-ELITE II. J Card Fail 1999; $5: 146-154$ the substantial lack of precision of the technology makes it unsuitable for prospective investigations. Despite its simplicity, the concept of QT dispersion does not offer a useful risk stratification of patients enrolled into large multicenter studies.

Future studies of repolarization abnormalities should focus on more precise measurements, not based on the duration of ECG intervals. ${ }^{16,24} \mathrm{~A}$ new evaluation of the $\mathrm{T}$ wave morphology from surface ECGs has been recently proposed. ${ }^{25}$ New morphological indices discriminated patients with hypertrophic cardiomyopathy from normal subjects more precisely than QT dispersion ${ }^{24,25}$ and selected indices of $\mathrm{T}$ wave morphology independently predicted adverse events during follow-up in postmyocardial infarction patients. ${ }^{26}$

13. Malik M, Camm AJ. Mystery of QTc interval dispersion. Am J Cardiol 1997; 79:785-787.

14. Zabel M, Franz MR, Klingenheben T, et al. Rate-dependence of QT dispersion and the QT interval: Comparison of atrial pacing and exercise testing. J Am Coll Cardiol 2000; 36:1654-1658.

15. Hnatkova K, Malik M, Kautzner J, et al. Adjustment of QT dispersion assessed from 12-lead electrocardiograms for different number of analysed electrocardiographic leads: Comparison of stability of different methods. Br Heart J 1994; 72:390-396.

16. Malik M, Batchvarov VN. Measurement, interpretation and clinical potential of QT dispersion. J Am Coll Cardiol 2000; 36:17491766.

17. Macfarlane PW, McLaughlin SC, Rodger JC. Influence of lead selection and population on automated measurement of QT dispersion. Circulation 1998; 98:2160-2167.

18. Kautzner J, Gang Y, Camm AJ, et al. Short and long term reproducibility of QT, QTc, and QTc dispersion measurement in healthy subjects. PACE 1994; 17:928-937.

19. Savelieva I, Gang Y, Guo X, et al. Agreement and reproducibility of automatic versus manual measurement of QT interval and QT dispersion. Am J Cardiol 1998; 81:471-477.

20. Fei L, Goldman JH, Prasad K, et al. QT dispersion and RR variations on 12-lead ECGs in patients with congestive heart failure secondary to idiopathic dilated cardiomyopathy. Eur Heart J 1996; 17:258263.

21. Brooksby P, Robinson PJ, Segal R, et al. Effects of losartan and captopril on QT dispersion in elderly patients with heart failure. ELITE study group. Lancet 1999; 354:395-396.

22. van de Loo A, Arendts W, Hohnloser SH. Variability of QT dispersion measurements in the surface electrocardiogram in patients with acute myocardial infarction and in normal subjects. Am J Cardiol 1994; 74:1113-1118.

23. Murray A, McLaughlin NB, Bourke JP, et al. Errors in manual measurement of QT intervals. Br Heart J 1994; 71:386-390.

24. Malik M, Acar B, Gang Y, et al. QT dispersion does not represent electrocardiographic interlead heterogeneity of ventricular repolarization. J Cardiovasc Electrophysiol 2000; 11:835843.

25. Acar B, Gang Y, Hnatkova K, et al. Spatial, temporal and wavefront direction characteristics of 12 lead T wave morphology. Medical Biol Eng Comput 1999; 37:574-584.

26. Zabel M, Acar B, Klingenheben T, et al. Analysis of 12-lead Twave morphology for risk stratification after myocardial infarction. Circulation 2000; 102:1252-1257. 